\title{
Prescribing trends before and after implementation of an antimicrobial stewardship program
}

\section{Kelly A Cairns \\ BPharm, Antimicrobialsherm, Stewardship
Pharmacist}

Adam W J Jenney

MB BS, FRACP, FRCPA

Infectious Diseases

Physician and

Microbiologist ${ }^{1,2}$

lain J Abbott

MB BS,

Microbiology Registrar

Matthew J Skinner

MB BS, FRACP

General Physician ${ }^{1,3}$

Joseph S Doyle

MB BS, MSC, FRACP

Infectious Diseases

Physician

Michael Dooley

BPharm,

Director of Pharmacy,' and

Professor of Clinical

Pharmacy, Centre for Medication Use and Safety ${ }^{2}$

Allen C Cheng

$\mathrm{MPH}, \mathrm{PhD}, \mathrm{FRACP}$

Deputy Head, Infection

Prevention and Hospital

Epidemiology Unit,' and

Associate Professor of

Infectious Diseases

Epidemiology²

1 Alfred Health Melbourne, VIC

2 Monash University Melbourne, VIC

3 Sir Charles

Gairdner Hospital

Perth, WA.

allen.cheng@

monash.edu

MJA 2013; 198: 262-266 doi: 10.5694/mjal2.11683 $\mathrm{p}$ to $50 \%$ of antimicrobial agents prescribed to hospital inpatients are considered to be inappropriate, $^{1,2}$ and this excess use has been associated with increased mortality, adverse drug reactions and the development of resistant bacteria. ${ }^{3,4}$ The Australian Commission on Safety and Quality in Health Care recently published recommendations for hospital-based antimicrobial stewardship programs. ${ }^{2}$ A variety of approaches are available to implement these recommendations, including dissemination of guidelines, education, restricting antimicrobial availability and postprescribing audit and review.

We aimed to evaluate changes in antimicrobial prescribing after the implementation of an antimicrobial stewardship program in a specialist tertiary referral hospital.

\section{Methods}

\section{Setting}

Alfred Health is a health service comprising three hospitals in metropolitan Melbourne. The largest campus, the Alfred Hospital, is a 430-bed tertiary teaching hospital with medicine, surgery and trauma services. It includes immunocompromised populations (including patients with HIV, cystic fibrosis and heart/lung transplantation, and haematology and bone marrow transplantation) and is supported by a 35-bed intensive care unit (ICU).

\section{Antimicrobial stewardship program}

We have previously described the preliminary activities of the antimicrobial stewardship team. ${ }^{5}$ A web-based antimicrobial approval system (Guidance MS, Melbourne Health) was rolled out from October $2010^{6}$ and a

\section{Abstract}

Objectives: Antimicrobial stewardship programs are recommended to reduce antimicrobial resistance by reducing inappropriate use of antimicrobials. We implemented an antimicrobial stewardship program and aimed to evaluate its effect on broad-spectrum antimicrobial use.

Design, setting and participants: Observational study with historical control using interrupted time series analysis conducted in a tertiary referral hospital. Hospital inpatients prescribed restricted antimicrobials for non-standard indications, where approval had expired or without approval.

Intervention: Baseline period of 30 months immediately followed by an 18-month intervention period commencing January 2011.

Main outcome measures: Number and type of interventions made by antimicrobial stewardship team; monthly rate of use of broad-spectrum antimicrobial agents (in defined daily doses/1000 occupied bed-days).

Results: The antimicrobial stewardship team made 1104 recommendations in 779 patients during the 18 -month intervention period. In $64 \%$ of cases, the recommendation was made to cease or de-escalate the antimicrobial therapy, or to change from intravenous to oral therapy. The introduction of the intervention resulted in an immediate $17 \%(95 \% \mathrm{Cl}, 13 \%-20 \%)$ reduction in broad-spectrum antimicrobial use in the intensive care unit and a $10 \%(95 \% \mathrm{Cl}$, $4 \%-16 \%$ ) reduction in broad-spectrum antimicrobial use outside the intensive care unit. Reductions were particularly seen in cephalosporin and glycopeptide use, although these were partially offset by increases in the use of $\beta$-lactam- $\beta$ lactamase inhibitors.

Conclusions: The introduction of an antimicrobial stewardship program, including postprescription review, resulted in an immediate reduction in broadspectrum antimicrobial use in a tertiary referral centre. However, the effect of this intervention reduced over time.

full-time pharmacist was appointed in January 2011. Before this, authorisation to prescribe restricted antimicrobial agents required approval from infectious diseases (ID) registrars, but auditing had suggested poor compliance. In the new system, online approval could be obtained to use restricted antimicrobials for preapproved indications that were included in national or local consensus guidelines. Short-term approval was granted for other indications specified by the clinician (non-standard indications). Pharmacists could alert the antimicrobial stewardship team of unauthorised antimicrobial use exceeding 24 hours (pharmacist alerts).

Non-ICU antimicrobial stewardship ward rounds (by the stewardship phar- macist and either an ID registrar and/ or an ID physician, on weekdays) commenced in January 2011. Each round comprised a focused review of clinical notes and results of investigations aimed at establishing the indication, planned duration, appropriateness, and alternatives to the use of restricted antimicrobial agents. Recommendations were discussed with the treating team and documented in writing; the final decision regarding patient management was the responsibility of the treating team. Patients who required more in-depth management advice were referred to the ID consult service.

Patients were reviewed by the stewardship team if they were receiving at least one restricted antimicrobial for a non-standard indication, where approval had expired, or 


\section{Existing infectious diseases services and antimicrobial stewardship interventions introduced during the study}

Existing infectious diseases services

Antimicrobial stewardship interventions introduced during this study

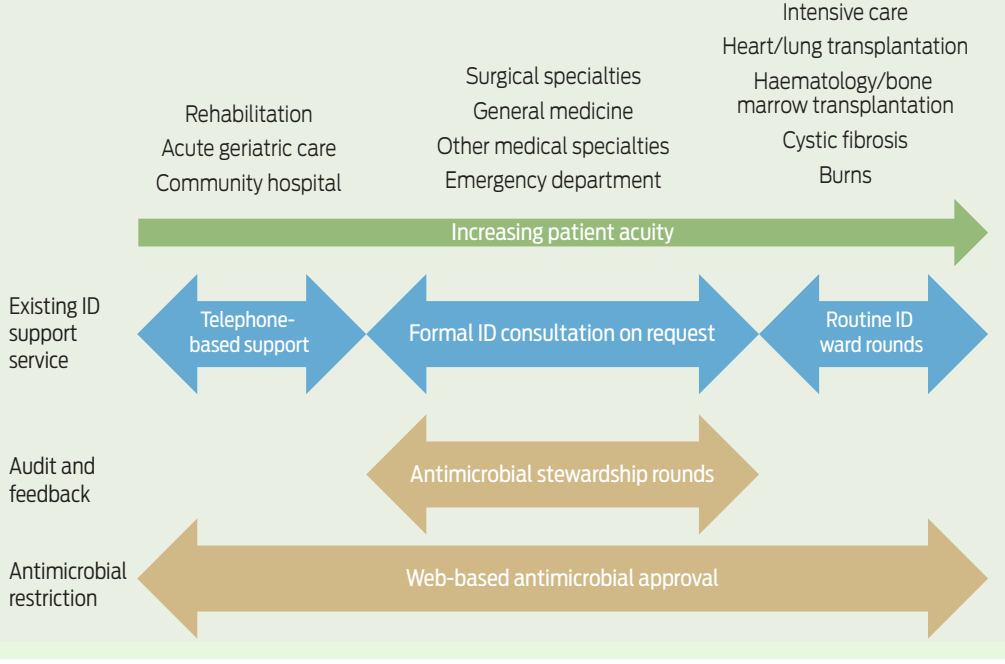

where a pharmacist alert had been created. At our hospital, 13 restricted antimicrobial agents require webbased approval: amikacin, azithromycin, cefepime, ceftazidime, ceftriaxone, ciprofloxacin, meropenem, moxifloxacin, piperacillin/tazobactam, teicoplanin, ticarcillin/clavulanate, tobramycin and vancomycin. Patients were not reviewed by the antimicrobial stewardship team if they had already received a formal ID consult, or were admitted under lung transplant/ cystic fibrosis, haematology and bone marrow transplant, or burns services, where ID physicians performed regular ward rounds (Box 1).

For several years in the ICU, the microbiology registrar has discussed results and antimicrobial treatments with ICU teams daily (supported by an ID physician three times per week). The stewardship pharmacist augmented this from January 2011 with all patients reviewed routinely. In December 2010, there was also a change to empirical ICU guidelines for health care-acquired sepsis, from ticarcillin/clavulanate or cefepime (for early and late sepsis, respectively) to piperacillin/tazobactam (regardless of onset), in all cases combined with an aminoglycoside, except when combined with quinolone in specified situations. Recommendations for vancomycin use did not change.

\section{Outcome measures}

We compared trends in the rate of use of antimicrobial classes before stewardship implementation (January 2008 to December 2010) and after implementation (January 2011 to June 2012). Antimicrobial consumption quantities were converted into defined daily doses (DDD) per 1000 occupied bed-days (OBD) as part of the National Antimicrobial Utilisation Surveillance Program. ${ }^{7,8}$ Total broadspectrum antimicrobial use was defined as the sum of usage for all classes except for aminoglycosides, which are regarded as narrow-spectrum antibiotics. Antimicrobial use is based on pharmacy purchasing data and inpatient stock distribution (excluding hospital in the home and

\section{Change in antimicrobial use before and after implementation of antimicrobial stewardship interventions}

\begin{tabular}{|c|c|c|c|c|c|c|c|}
\hline \multirow[b]{2}{*}{ Antimicrobial class/setting } & \multicolumn{2}{|c|}{ Before intervention } & \multicolumn{2}{|c|}{ After intervention } & \multicolumn{3}{|c|}{ Change } \\
\hline & Use* & $\begin{array}{c}\text { Trend } \\
(\% / \text { month })^{\dagger}\end{array}$ & Use* & $\begin{array}{c}\text { Trend } \\
(\% / \text { month })^{\dagger}\end{array}$ & $\begin{array}{l}\text { Change } \\
\text { in use }^{\dagger}\end{array}$ & $\begin{array}{l}\text { Immediate change } \\
(95 \% \mathrm{Cl})^{\ddagger}\end{array}$ & $\begin{array}{l}\text { Change in trend } \\
(95 \% \mathrm{Cl})^{\delta}\end{array}$ \\
\hline \multicolumn{8}{|l|}{ Intensive care } \\
\hline Total broad spectrum & 1021.8 & 0 & 937.1 & $1.0 \%$ & $-8.3 \%$ & $-16.6 \%(-19.9 \%,-13.2 \%)$ & $1.0 \%(0.7 \%, 1.4 \%)$ \\
\hline Aminoglycosides & 137.0 & $-2.0 \%$ & 75.2 & $-0.5 \%$ & $-45.1 \%$ & - 20.3\% (- 30.2\%, - 9.1\%) & $1.5 \%(0.4 \%, 2.7 \%)$ \\
\hline $\begin{array}{l}\text { Antipseudomonal } \beta \text {-lactam- } \\
\beta \text {-lactamase inhibitor }\end{array}$ & 129.1 & $0.3 \%$ & 191.3 & $0.6 \%$ & $48.2 \%$ & $34.2 \%(21.8 \%, 47.9 \%)$ & $0.3 \%(-0.5 \%, 1.1 \%)$ \\
\hline Carbapenems & 113.8 & $0.4 \%$ & 133.9 & $2.4 \%$ & $17.6 \%$ & $-11.2 \%$ (- 20.7\%, - 0.6\%) & $2.1 \%(1.2 \%, 3.0 \%)$ \\
\hline $\begin{array}{l}\text { Cephalosporins } \\
\text { (3rd/4th generation) }\end{array}$ & 219.2 & $0.8 \%$ & 131.2 & $1.6 \%$ & $-40.2 \%$ & $-54.6 \%(-59.0 \%,-49.7 \%)$ & $0.8 \%(-0.1 \%, 1.7 \%)$ \\
\hline Fluoroquinolones & 318.3 & $-0.7 \%$ & 278.4 & $0.1 \%$ & $-12.5 \%$ & $-3.3 \%(-10.1 \%, 4.0 \%)$ & $0.7 \%(0.1 \%, 1.4 \%)$ \\
\hline Glycopeptides & 241.4 & $-0.2 \%$ & 202.3 & $1.5 \%$ & $-16.2 \%$ & $-24.8 \%(-31.1 \%,-18.0 \%)$ & $1.7 \%(1.0 \%, 2.5 \%)$ \\
\hline \multicolumn{8}{|c|}{ General wards (excluding intensive care) } \\
\hline Total broad spectrum & 357.8 & $0.1 \%$ & 333.4 & $0.3 \%$ & $-6.8 \%$ & $-9.9 \%(-15.7 \%,-3.7 \%)$ & $0.2 \%(-0.4 \%, 0.8 \%)$ \\
\hline Aminoglycosides & 63.7 & $-1.0 \%$ & 55.8 & $-0.7 \%$ & $-12.5 \%$ & $9.8 \%(-6.7 \%, 29.1 \%)$ & $0.3 \%(-1.1 \%, 1.7 \%)$ \\
\hline $\begin{array}{l}\text { Antipseudomonal } \beta \text {-lactam- } \\
\beta \text {-lactamase inhibitor }\end{array}$ & 50.5 & $-0.4 \%$ & 54.5 & $1.9 \%$ & $8.1 \%$ & $-2.9 \%(-18.5 \%, 15.7 \%)$ & $2.3 \%(0.9 \%, 3.7 \%)$ \\
\hline Carbapenems & 52.9 & $-0.4 \%$ & 53.5 & $0.1 \%$ & $1.0 \%$ & $6.7 \%(-10.0 \%, 26.5 \%)$ & $0.5 \%(-0.9 \%, 2.0 \%)$ \\
\hline $\begin{array}{l}\text { Cephalosporins } \\
\text { (3rd/4th generation) }\end{array}$ & 90.1 & $0.5 \%$ & 80.3 & $0.7 \%$ & $-10.9 \%$ & $-22.4 \%(-32.3 \%,-11.1 \%)$ & $0.2 \%(-1.0 \%, 1.4 \%)$ \\
\hline Fluoroquinolones & 81.8 & 0 & 74.0 & $-0.6 \%$ & $-9.6 \%$ & $-4.2 \%(-16.7 \%, 10.3 \%)$ & $-0.6 \%(-1.8 \%, 0.7 \%)$ \\
\hline Glycopeptides & 82.5 & $0.3 \%$ & 71.2 & $-0.4 \%$ & $-13.8 \%$ & $-14.2 \%(-25.6 \%,-1.2 \%)$ & $-0.7 \%(-2.0 \%, 0.5 \%)$ \\
\hline
\end{tabular}

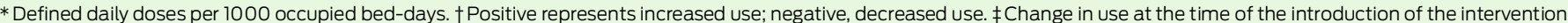

$\oint$ Relative change in monthly rate of use. 
the emergency department). Outcomes were assessed by:

- the mean rate of antimicrobial use in the intervention period compared with the pre-intervention period;

- model-predicted immediate change in antimicrobial use between the end of the preintervention period and the commencement of the intervention period (immediate change);

- model-predicted change in the rate of antimicrobial use between the pre-intervention period and postintervention period (change in trend);

- the immediate change and the change in trend in antimicrobial use were both assessed using segmented Poisson regression.

We defined a clinically significant decrease in antimicrobial use as:

- a statistically significant $(P<0.05)$ immediate decrease in the rate of antimicrobial use; and/or

- a statistically significant decrease in the rate of change of antimicrobial use in the intervention period compared with the pre-intervention period.

Statistical tests were performed using Stata version 12 (StataCorp). Ethical permission to review these data was obtained from the Alfred Health Human Ethics Committee.

\section{Results}

\section{Impact of antimicrobial stewardship rounds}

Between 10 January 2011 and 30 June 2012, 2254 patients were identified as requiring review by the antimicrobial stewardship team. An antimicrobial management recommendation was made in 779 of 2254 (35\%) patients, with a total of 1104 recommendations made. Of the patients for whom a recommendation was made, the median age was 66 years (range, 1698 years) and 503 (65\%) were male.

Recommendations were made in patients under 26 different treating units; 63\% (490/779) of patients were managed by surgical/trauma units and $37 \%(289 / 779)$ were medical patients. The median duration of antimicrobial therapy before review was 2 days (interquartile range, 1-4 days). The majority of recommendations were made following pharmacy alerts
3 Antimicrobial use before and after implementation of the antimicrobial stewardship ward rounds, by class of antimicrobial agent
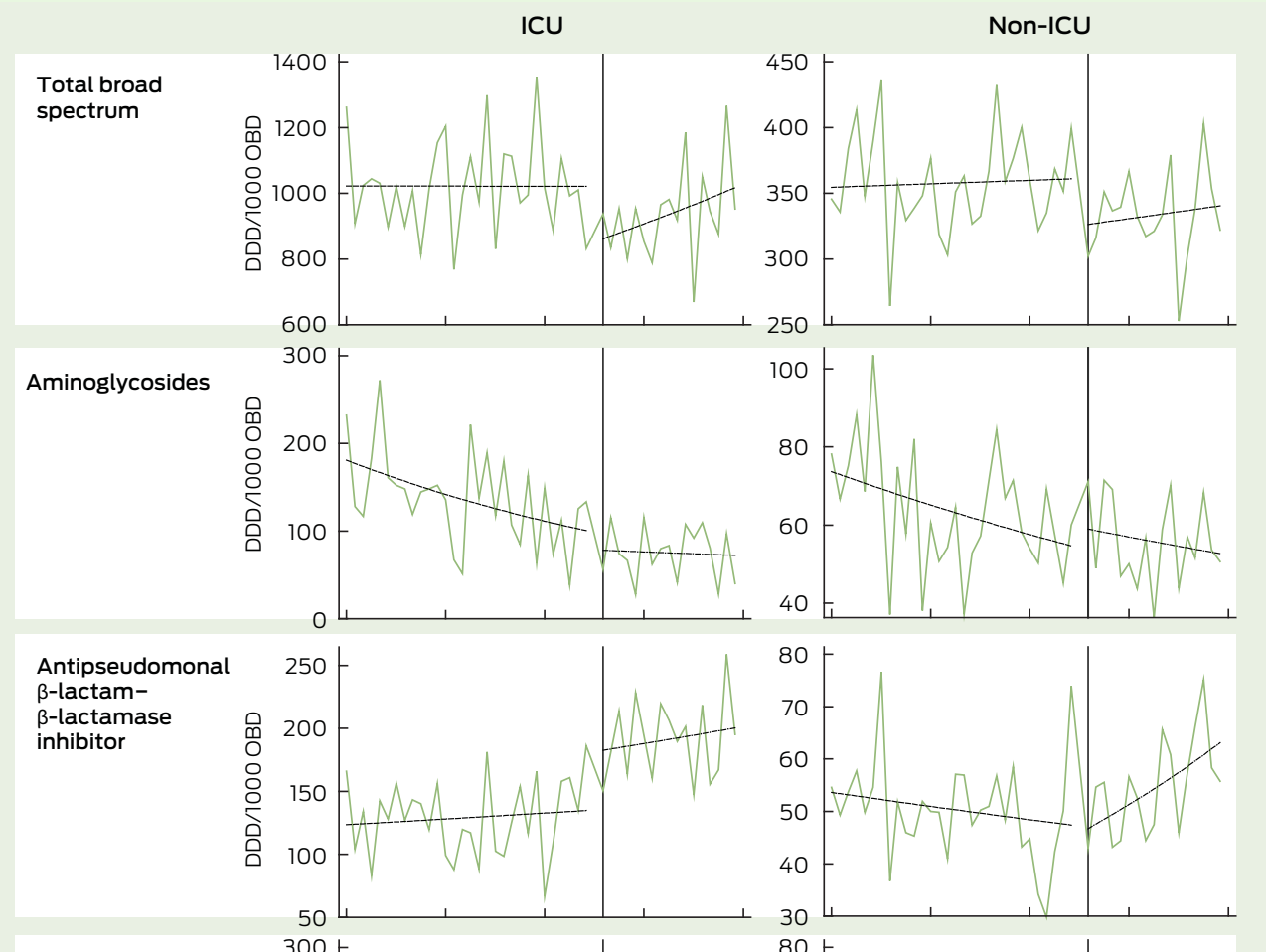

Carbapenems
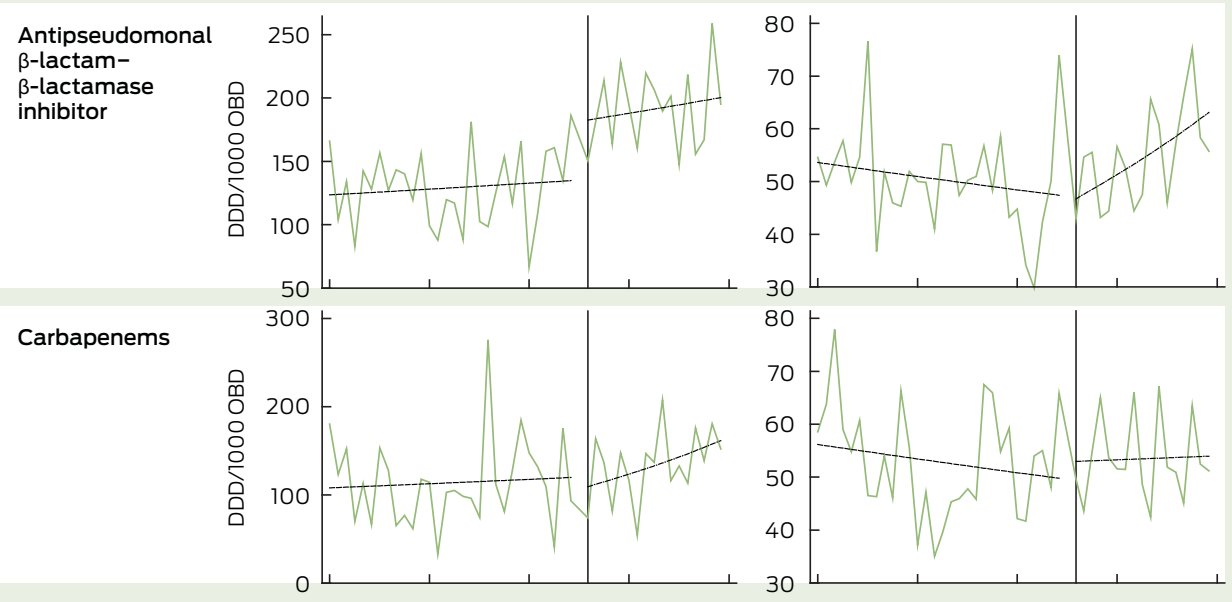

Cephalosporins
(3rd/4th
generation)
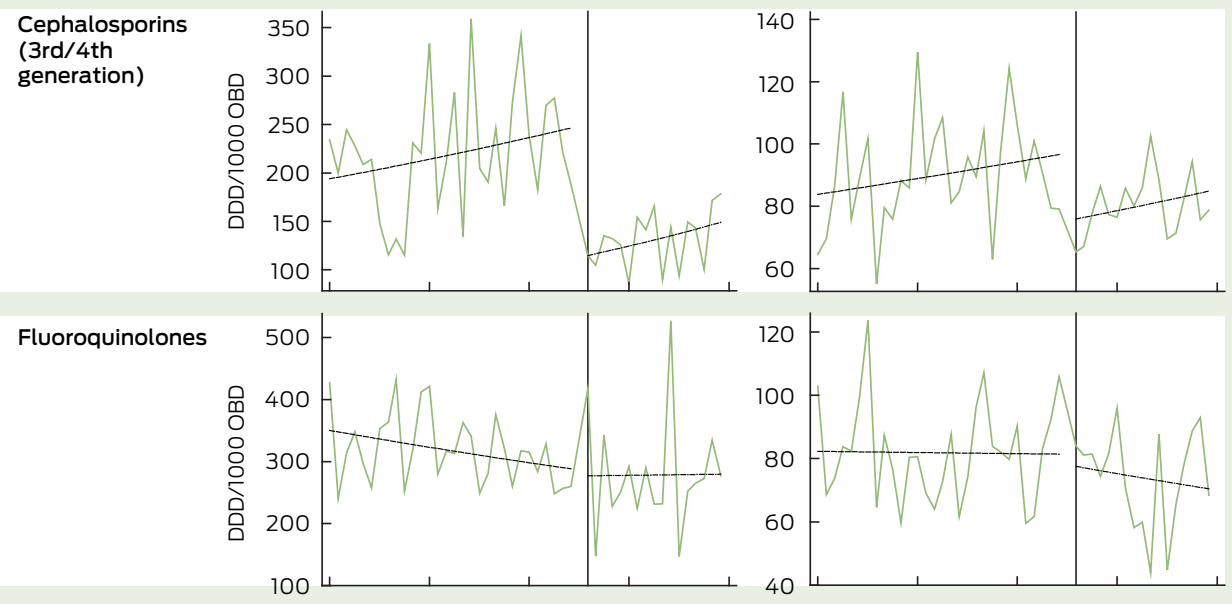

Glycopeptides
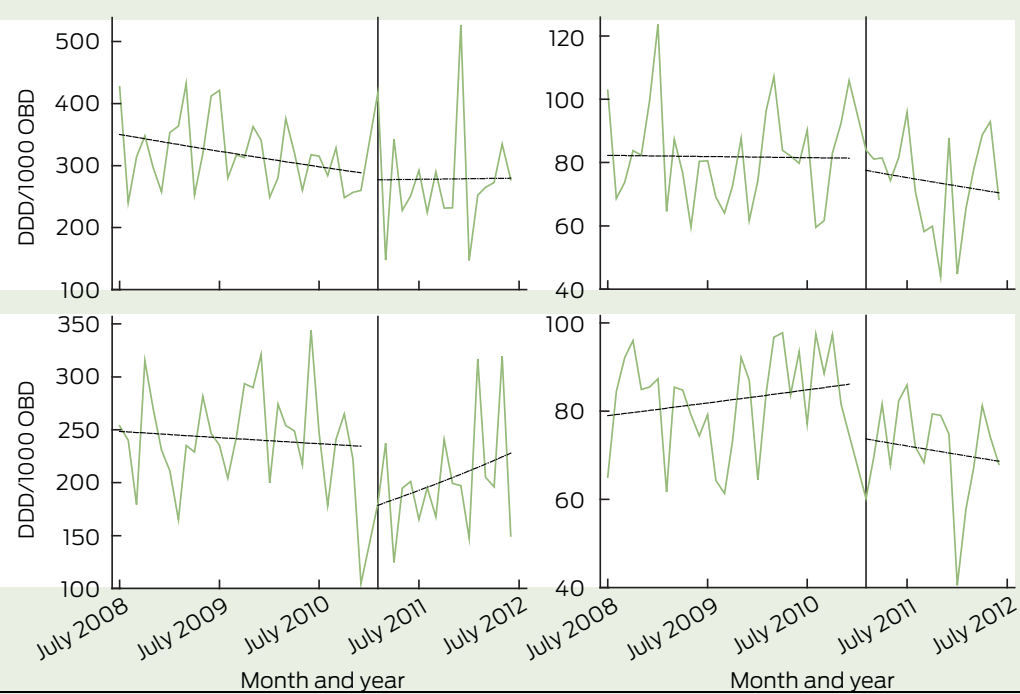

$\mathrm{DDD} / 1000 \mathrm{OBD}=$ defined daily doses per 1000 occupied bed-days. ICU = intensive care unit. Solid vertical line represents commencement of intervention. Dotted lines represent pre-intervention and post-intervention trends in antimicrobial use. 
(907/1104; 82\%), by non-standard approvals $(92 / 1104 ; 8 \%)$ or based on expiry of the current antimicrobial approval (93/1104; 8\%).

Recommendations were made to modify treatment for patients on restricted broad-spectrum antimicrobials; most commonly, ceftriaxone (278), piperacillin/tazobactam (155), ciprofloxacin (99) and vancomycin (96).

In $40 \%(440 / 1104)$ of recommendations, antimicrobial discontinuation was suggested; in an additional $11 \%$ (123/1104), antimicrobial deescalation was recommended; and in $13 \%(145 / 1104)$, an intravenous to oral switch was recommended. Escalation of antimicrobial spectrum was recommended in 2\% (25/1104) of cases and antimicrobial initiation in $3 \%$ (29/1104). A formal ID consult referral was recommended on 71 occasions (6\%).

In $74 \%(819 / 1104)$ of cases, the recommendation was accepted by the treating team. For most of the unaccepted recommendations (233/285; $82 \%)$, no reason was cited for nonacceptance. Where reasons for nonacceptance were documented, they included the use of unapproved unit protocols (13) and the insistence of a more senior doctor in the treating team (14).

\section{Impact on overall antimicrobial use}

In the ICU, total broad-spectrum antimicrobial use decreased immediately by $16.6 \%$ when the intervention commenced $(P<0.001)$ (Box 2$)$. The mean total use of broad-spectrum antimicrobials fell from 1022 DDD/1000 OBD in the pre-intervention period to 937 DDD/1000 OBD in the postintervention period. Before the intervention, the rate of broad-spectrum antimicrobial use did not change; following the intervention, it increased by $1.0 \%$ per month $(P<0.001)$. Changes in the use of specific classes of antimicrobials are detailed in Box 2 and Box 3 .

In hospital wards other than the ICU, total broad-spectrum antimicrobial use decreased by $9.9 \%$ when the intervention commenced $(P=0.002)$. The mean total use of broad-spectrum antimicrobials fell from 358 DDD/ $1000 \mathrm{OBD}$ in the pre-intervention period to $333 \mathrm{DDD} / 1000 \mathrm{OBD}$ in the post-intervention period. Before the intervention, the rate of broad-spectrum antimicrobial use increased by $0.1 \%$ per month; following the intervention, it increased by $0.3 \%$ per month $(P=0.49)$. Changes in the use of specific classes of antimicrobials are detailed in Box 2 and Box 3.

\section{Discussion}

The antimicrobial stewardship program brought immediate reductions in the use of total broad-spectrum antimicrobials, particularly third/fourth generation cephalosporins and glycopeptides. In addition to case-by-case audit and feedback, regular stewardship rounds identified unapproved unit guidelines, provided an accessible clinical resource for junior doctors, raised awareness of appropriate antimicrobial use and reinforced the use of the web-based antimicrobial approval system. Our experience is consistent with a systematic review of stewardship programs that suggested that restrictive interventions were more likely to be successful than those based only on education or persuasion. ${ }^{9}$

The interventions that we have implemented are resource intensive, requiring a full-time pharmacist supported by part-time ID physicians (810 hours/week). Although a previous study has shown a decrease in several classes of broad-spectrum antimicrobials associated with a web-based approval system only, ${ }^{6}$ we felt that without an audit and feedback mechanism, this intervention would not be sustainable. Additionally, postprescribing audit and feedback recognises that appropriateness of therapy often needs to be considered on a case-by-case basis, and that broad guidelines on prescribing may not be easily applied to individual patients. Previous studies of similar interventions have found similar patterns of intervention, but on a much less intensive scale. ${ }^{10-12}$ Despite this, only six of 78 respondents in an Australian survey of hospital pharmacies reported implementing regular multidisciplinary antimicrobial stewardship ward rounds. ${ }^{13}$

There are several limitations to this observational study. We were unable to definitively ascribe changes in prescribing to the intervention, due to confounders such as concurrent changes in ICU empirical treatment guidelines. Aggregated data on antimicrobial use is not able to provide a measure of appropriateness of use and does not account for changes in antimicrobial dosing. The data on antimicrobial use includes units known to be high users of broadspectrum antimicrobials (eg, cystic fibrosis) but where the only new intervention was the introduction of the web-based approval system. A formal cost-effectiveness study was not undertaken; however, we note that the antimicrobial classes where significant decreases in use were seen are relatively inexpensive (ceftriaxone $1 \mathrm{~g}, \$ 1$; vancomycin $1 \mathrm{~g}, \$ 3$ ) and thus are unlikely to offset the cost of the stewardship team based on saved drug costs alone. The antimicrobial use data used in this study were based on pharmacy purchasing data and inpatient stock distribution, with purchasing practices likely to have affected use data and to have potentially introduced delays in use trends. A 2-month worldwide benzylpenicillin shortage occurred during the study period (September-November 2011), which may have affected antimicrobial use trends at this time.

We attempted to reduce potential adverse effects by using built-in safeguards, including the provision to commence antimicrobials without approval for 24 hours, routinely discussing recommendations with the clinical team, and leaving the final decision regarding changes to antimicrobial therapy to the treating clinicians. We found evidence of greater use of $\beta$-lactam- $\beta$-lactamase inhibitor combinations that offset the decreased use of other classes, particularly cephalosporins and aminogylcosides - a phenomenon termed "squeezing the antibiotic balloon". Concerningly, in the ICU we found some evidence of a rebound in the overall use of antimicrobials, and specifically, in the use of carbapenems, fluoroquinolones and glycopeptides. Further work is required to improve the quality of prescribing and evaluate longer term effects on antimicrobial resistance and patient outcomes.

Acknowledgements: We acknowledge the previous antimicrobial stewardship pharmacists involved in this project (Jenny Kirschner, Trent Lee) and the members of the Alfred Health Antimicrobial Stewardship Committee. 
We also thank Alex Padiglione for helpful comments on the manuscript, and the National Antibiotic Utilisation Surveillance Program for data.

Competing interests: No relevant disclosures. Received 16 Nov 2012, accepted 17 Feb 2013.

1 Gottlieb T, Nimmo GR. Antibiotic resistance is an emerging threat to public health: an urgent call to action at the Antimicrobial Resistance Summit 2011. Med J Aust 2011; 194: 281-283.

2 Duguid M, Cruickshank M. Antimicrobial stewardship in Australian hospitals. Sydney: Australian Commission on Safety and Quality in Healthcare, 2011.

3 Dellit TH, Owens RC, McGowan JE Jr, et al. Infectious Diseases Society of America and the Society for Healthcare Epidemiology of America guidelines for developing an institutional program to enhance antimicrobial stewardship. Clin Infect Dis 2007; 44: 159-177.

4 Gums JG, Yancey RW Jr, Hamilton CA, Kubilis PS. A randomized, prospective study measuring outcomes after antibiotic therapy intervention by a multidisciplinary consult team.

Pharmacotherapy 1999; 19: 1369-1377.
5 Cairns KA, Jenney AWJ, Krishnaswamy S, et al. Early experience with antimicrobial stewardship ward rounds at a tertiary referral hospital [letter] Med J Aust 2012; 196: 34-35.

6 Buising KL, Thursky KA, Robertson MB, et al. Electronic antibiotic stewardship - reduced consumption of broad-spectrum antibiotics using a computerized antimicrobial approval system in a hospital setting. J Antimicrob Chemother 2008; 62: 608-616.

7 Natsch S, Hekster YA, de Jong R, et al. Application of the ATC/DDD methodology to monitor antibiotic drug use. Eur J Clin Microbiol Infect Dis 1998; 17: 20-24.

8 South Australian Infection Control Service. National Antimicrobial Utilisation Surveillance Program: annual report 2010-2012. http:// www.health.sa.gov.au/INFECTIONCONTROL/ Default.aspx?PageContentID=65\&tabid $=199$ (accessed Nov 2012).

9 Davey P, Brown E, Fenelon L, et al. Interventions to improve antibiotic prescribing practices for hospital inpatients. Cochrane Database Syst Rev 2005; (4): CD003543.
10 Baker CA, Sehdev P. Defining an antimicrobial monitoring service. Am J Health Syst Pharm 2008; 65: 2095-2096, 2098-2099.

11 Laible BR, Nazir J, Assimacopoulos AP, Schut J. Implementation of a pharmacist-led antimicrobial management team in a community teaching hospital: use of pharmacy residents and pharmacy students in a prospective audit and feedback approach. J Pharm Pract 2010; 23: 531-535.

12 Cosgrove SE, Seo SK, Bolon MK, et al. Evaluation of postprescription review and feedback as a method of promoting rational antimicrobial use: a multicenter intervention. Infect Control Hosp Epidemiol 2012; 33: 374-380.

13 Chen AWJ, Khumra S, Eaton V, Kong DCM. Snapshot of antimicrobial stewardship in Australian hospitals. J Pharm Prac Res 2010; 40: 19-26.

14 Peterson LR. Squeezing the antibiotic balloon: the impact of antimicrobial classes on emerging resistance. Clin Microbiol Infect 2005; 11 Suppl 5: 4-16. 